\title{
DECOMPOSITION ALGEBRAS OF RIESZ OPERATORS
}

\author{
by G. J. MURPHY and T. T. WEST
}

(Received 11 December, 1978)

Let $H$ be a Hilbert space and let $\mathbf{B}$ denote the Banach algebra of all bounded linear operators on $H$ with $\mathbf{K}$ denoting the closed ideal of compact operators in $\mathbf{B}$. If $T \in \mathbf{B}, \sigma(T)$ and $r(T)$ will denote the spectrum and spectral radius of $T$, respectively, and $\pi$ the canonical mapping of $\mathbf{B}$ onto the Calkin algebra $\mathbf{B} / \mathbf{K}$.

$R \in \mathbf{B}$ is called a Riesz operator if $\pi(R)$ is a quasinilpotent element of the Calkin algebra. The second author [9] has proved that every Riesz operator $R=C+Q$ where $C$ is compact normal, $Q$ is quasinilpotent and $\sigma(R)=\sigma(C)$. It follows from a theorem of Ruston [8] that this decomposition characterises Riesz operators on Hilbert spaces and it is an open problem whether such a decomposition is possible in Banach spaces. Similar decompositions have occurred in the work of Gohberg and Krein [3, p. 17] and Stampfli [7, Lemma 6]. As there may be many different decompositions of a Riesz operator into the sum of a compact and a quasinilpotent operator we shall call the decomposition as carried out in [9] and described below a West decomposition. There can be more than one West decomposition of the same Riesz operator.

Gillespie and West [2] have given an example of a Riesz operator on a Hilbert space which cannot be decomposed in any manner so that the compact and quasinilpotent parts commute. From now on we consider a fixed West decomposition $R=C+Q$. Chui, Smith and Ward [1] have proved that $N=C Q-Q C$ is quasinilpotent and R. E. Harte has remarked that $p(C, Q) N$ is quasinilpotent for any polynomial $p$ in two non-commuting variables. This prompted an investigation of the (in general non-unital) closed subalgebra of B generated by $C$ and $Q$ which we call the decomposition algebra of $R$ associated with $C$ and $Q$ and denote by $\mathbf{D}(C, Q)$ or, when there is no possibility of ambiguity, by $\mathbf{D}$. We show that $Q$ and $N$ are both contained in $\operatorname{Rad} \mathbf{D}$ the radical of $\mathbf{D}$ which consists of all the quasinilpotent elements of $\mathbf{D}$, that $\mathbf{D}$ is commutative modulo its radical, that $\mathbf{D}$ consists entirely of Riesz operators and thus is a Riesz algebra in the sense of Smyth [6], that the quotient algebra $\mathbf{D} / \operatorname{Rad} \mathbf{D}$ is invariant for all West decompositions of $R$ and that $\mathbf{D}$ is the direct sum of $\operatorname{Rad} \mathbf{D}$ and the closed subalgebra generated by $C$.

Smyth [5] has successfully put Riesz theory in an algebraic setting and has obtained a perfect analogue of the West decomposition for Riesz elements of $\mathrm{C}^{*}$-algebras. Legg [4] has shown that the result of Chui, Smith and Ward holds in this context; similarly all the results proved here extend easily to the $C^{*}$-algebra setting.

We shall need the facts that the spectral radius is continuous relative to the norm topology for operators in a commutative algebra or if the spectrum of the limit operator is totally disconnected (as is the case for Riesz operators). Also if $A \in \mathbf{B}$ can be written as

Glasgow Math. J. 21 (1980) 75-79. 
the operator matrix, relative to some decomposition of $H$,

$$
A=\left[\begin{array}{cc}
A_{11} & A_{12} \\
0 & A_{22}
\end{array}\right]
$$

then $\sigma(A) \subseteq \sigma\left(A_{11}\right) \cup \sigma\left(A_{22}\right)$.

Let $R$ be a Riesz operator on $H$. To avoid trivialities we assume that $H$ is infinite dimensional and that $\sigma(R)=\left\{\lambda_{i}\right\}_{1}^{\infty} \cup\{0\}$, where $\left\{\lambda_{j}\right\}_{1}^{\infty}$ is the set of non-zero eigenvalues of $R$. It is well known that $\lambda_{j} \rightarrow 0(j \rightarrow \infty)$. Let $P_{j}$ denote the spectral projection for $R$ corresponding to the eigenvalue $\lambda_{j}$ given by the formula

$$
P_{i}=\frac{1}{2 \pi i} \int_{\Gamma_{i}}(z I-R)^{-1} d z
$$

where $\Gamma_{j}$ is a circle centre $\lambda_{j}$ containing no other eigenvalue of $R$ in its interior or on its boundary. Put $S_{k}=\sum_{i=1}^{k} P_{j}$, and $F_{k}$ for the orthogonal projection whose range is the same as that of $S_{k}$. Write $E_{j}=F_{j}-F_{j-1}(j=1,2, \ldots)$ with $F_{0}=0$. Then $C=\sum_{j=1}^{\infty} \lambda_{j} E_{j}$ is the compact normal operator in the West decomposition of $R$ (relative to this arrangement of the eigenvalues) and the essence of the proof lies in showing that $Q=R-C$ is quasinilpotent. If $C_{k}=\sum_{j=1}^{k} \lambda_{i} E_{j}$ and we consider $H$ as the orthogonal direct sum of the range and null-space of the projection $F_{k}, C_{k}$ and $Q$ may be written in matrix form

$$
C_{k}=\left[\begin{array}{cccc|c}
\lambda_{1} & & & 0 & 0 \\
& \lambda_{2} & & & \\
& & \lambda_{3} & & \\
& & & \lambda_{k} & \\
\hline & 0 & & 0
\end{array}\right], \quad Q=\left[\begin{array}{cccc|c}
0 & & & * & * \\
& 0 & & & \\
& & 0 & & \\
& & 0 & 0 & \\
\hline & & & 0 & *
\end{array}\right],
$$

where in the $C_{k}$ matrix the eigenvalues are repeated according to multiplicity. Note that we have essentially chosen a basis of the range of each of the $E_{j}$ 's (up to $j=k$ ) which upper-triangularises the matrices for $C, R$ and $Q$.

Lemma 1 (Harte). $p(C, Q) N$ is quasinilpotent, where $p(C, Q)$ is any polynomial in $C$ and $Q$.

Proof. Put $N_{k}=C_{k} Q-Q C_{k}$. Then $C_{k} \rightarrow C(k \rightarrow \infty)$ hence

$$
p\left(C_{k}, Q\right) N_{k} \rightarrow p(C, Q) N \quad(k \rightarrow \infty) .
$$

$N$ is compact and therefore

$$
r\left\{p\left(C_{k}, Q\right) N_{k}\right\} \rightarrow r\{p(C, Q) N\} \quad(k \rightarrow \infty) .
$$


It suffices to show that $p\left(C_{k}, Q\right) N_{k}$ is quasinilpotent for each $k$. Now

$$
\begin{gathered}
N_{k}=\left[\begin{array}{lll|l}
0 & & * & * \\
& 0 & & \\
& 0 & 0 & \\
\hline 0 & & 0
\end{array}\right], \quad p\left(C_{k}, Q\right)=\left[\begin{array}{lll|l}
\mu_{1} & & * & * \\
& \mu_{2} & \\
0 & & \\
\hline & 0 & *
\end{array}\right], \\
p\left(C_{k}, Q\right) N_{k}=\left[\begin{array}{lll|l}
0 & & & \\
& 0 & * & * \\
& 0 & 0 & \\
\hline 0 & & 0
\end{array}\right]
\end{gathered}
$$

and the result follows as the matrix is nilpotent.

Proposition 2. $N \in \operatorname{Rad} D$.

Proof. It suffices to show that $r(T N)=0$ for each $T \in \mathbf{D}$. Now there exists a sequence of polynomials $p_{n}(C, Q)$ converging to $T$ as $n \rightarrow \infty$. Thus

$$
p_{n}(C, Q) N \rightarrow T N \quad(n \rightarrow \infty)
$$

and $N$ is compact; hence

$$
r\left\{p_{n}(C, Q) N\right\} \rightarrow r(T N) \quad(n \rightarrow \infty) .
$$

The result follows from Lemma 1.

Corollary 3. $\mathbf{D}^{\prime}=\mathbf{D} / \operatorname{Rad} \mathbf{D}$ is commutative.

Proposition 4. Rad D consists of the quasinilpotent elements of $\mathbf{D}$.

Proof. Every operator in $\operatorname{Rad} \mathbf{D}$ is quasinilpotent. By Corollary 3 and the results of [10] the spectral radius is subadditive and submultiplicative on $\mathbf{D}$; hence the quasinilpotents form an ideal of $\mathbf{D}$ which is therefore contained in $\operatorname{Rad} \mathbf{D}$.

Corollary 5. $Q \in \operatorname{Rad} \mathbf{D}$.

Proposition 6. Every element of $\mathbf{D}$ is a Riesz operator.

Proof. If $T \in \mathbf{D}$ there is a sequence of polynomials $p_{n}(C, Q)$ without constant term converging to $T$. Then

$$
\pi\left(p_{n}(C, Q)\right)=p_{n}(0, \pi(Q)) \rightarrow \pi(T) \quad(n \rightarrow \infty) .
$$


$\pi(Q)$ is quasinilpotent and $p_{n}(0, \pi(Q))$ is a polynomial in $\pi(Q)$ without a constant term; thus $\pi(T)$ is contained in the commutative subalgebra generated by $\pi(Q)$; hence $r\left\{p_{n}(0, \pi(Q))\right\} \rightarrow r(\pi(T))(n \rightarrow \infty)$ and $r(\pi(T))=0$.

Proposition 7. $\mathbf{D}^{\prime}$ is isometrically isomorphic to the closed subalgebra $\mathbf{A}(C)$ of $\mathbf{B}$ generated by $C$, and is thus independent of the particular West decomposition of $R$.

Proof. $\mathbf{D}^{\prime}$ is a commutative semi-simple Banach algebra generated by $C^{\prime}=$ $C+\operatorname{Rad} \mathbf{D}$. Now $\sigma_{\mathbf{D}^{\prime}}\left(T^{\prime}\right)=\sigma_{\mathbf{D}}(T)(T \in \mathbf{D})$, and so $r\left(T^{\prime}\right)=r(T)=\|T\|$ if $T \in \mathbf{A}(C)$ (as $C$ is normal); hence $\|T\|=\left\|T^{\prime}\right\|$. Thus the map $\mathbf{A}(C) \rightarrow \mathbf{D}^{\prime}: T \rightarrow T^{\prime}$ is an isometric isomorphism. If $R=C_{1}+Q_{1}$ is a second West decomposition, then there is a unitary operator $U$ for which $C=U C_{1} U^{-1}$; thus $\mathbf{A}(C)$ and $\mathbf{A}\left(C_{1}\right)$ are isometrically isomorphic.

Proposition 8. $\mathbf{D}=\mathbf{A}(C) \oplus \operatorname{Rad} \mathbf{D}$.

Proof (i). If $T \in \mathbf{A}(C) \cap \operatorname{Rad} \mathbf{D}$, then $T$ is both normal and quasinilpotent and hence zero.

(ii) If $T \in \mathbf{D}$ there exists a sequence of polynomials $p_{n}(C, Q)$ converging to $T$. Thus

$$
p_{n}(C, Q)^{\prime}=p_{n}\left(C^{\prime}, 0\right) \rightarrow T^{\prime} \quad(n \rightarrow \infty) .
$$

By Proposition 7, as $n \rightarrow \infty, \quad p_{n}(C, 0) \rightarrow S \in \mathbf{A}(C)$ and $S^{\prime}=T^{\prime}$. Thus, as $n \rightarrow \infty$, $p_{n}(C, Q)-p_{n}(C, 0) \rightarrow S-T \in \operatorname{Rad} \mathbf{D}$ and hence $\mathbf{D}=\mathbf{A}(C)+\operatorname{Rad} \mathbf{D}$, completing the proof.

These results fail to hold for decompositions of Riesz operators which are not West decompositions. If

$$
C=\left[\begin{array}{ll}
1 & 0 \\
0 & 0
\end{array}\right], \quad Q=\left[\begin{array}{rr}
1 & 1 \\
-1 & -1
\end{array}\right]
$$

then $C$ is compact normal, and $Q$ is quasinilpotent but they have no proper invariant subspace in common. Thus the identity representation of the algebra generated by $C$ and $Q$ is irreducible and hence the algebra is semi-simple.

\section{REFERENCES}

1. C. K. Chui, P. W. Smith and J. D. Ward, A note on Riesz operators, Proc. Amer. Math. Soc. 60 (1976), 92-94.

2. T. A. Gillespie and T. T. West, A characterisation and two examples of Riesz operators, Glasgow Math. J. 9 (1968), 106-110.

3. I. C. Gohberg and M. G. Krein, Introduction to the theory of linear nonselfadjoint operators Vol. 18 Translations of Math. Monographs A.M.S. (Providence, 1969).

4. D. Legg, A note on Riesz elements in $C^{*}$-algebras, Internat. J. Math. and Math. Sci. 1 (1978), 93-96.

5. M. R. F. Smyth, Riesz theory in Banach algebras, Math. Z. 145 (1975), 145-155.

6. M. R. F. Smyth, Riesz algebras, Proc. Roy. Irish. Acad. Sect. A 76 (1976), 327-334.

7. J. G. Stampfli, Compact perturbations, normal eigenvalues and a problem of Salinas, $J$. London Math. Soc. (2), 9 (1974), 165-175.

8. T. T. West, Riesz operators in Banach spaces, Proc. London Math. Soc. (3) 16 (1966), 131-140. 
9. T. T. West, The decomposition of Riesz operators, Proc. London Math. Soc. (3) 16 (1966), 737-752.

10. J. Zemánek, Spectral radius characterizations of commutativity in Banach algebras. Studia Math. 61 (1977), 257-268.

School of Mathematics

Trinity College

DUBLIN 